\title{
Deterioro cognoscitivo y factores asociados en adultos mayores en México
}

\author{
Silvia Mejía-Arango, PhD, ${ }^{(1)}$ Alejandro Miguel-jaimes, MD, ${ }^{(1)}$ Antonio Villa, MD, (I) \\ Liliana Ruiz-Arregui, M en C, (I) Luis Miguel Gutiérrez-Robledo, MC, PhD. (I)
}

\author{
Mejía-Arango S, Miguel-Jaimes A, Villa A, \\ Ruiz-Arregui L, Gutiérrez-Robledo LM. \\ Deterioro cognoscitivo y factores asociados \\ en adultos mayores en México. \\ Salud Publica Mex 2007;49 supl 4:S475-S48I.
}

\section{Resumen}

Objetivo. Reportar la prevalencia de deterioro cognoscitivo en el adulto mayor y su relación con factores sociodemográficos y de salud en la población mexicana. Material y métodos. Del Estudio Nacional sobre Salud y Envejecimiento en México (ENASEM), realizado en el año 2001 en toda la República Mexicana, se seleccionaron todos los sujetos mayores de 65 años que hubieran completado la evaluación cognoscitiva, los cuales se clasificaron en dos grupos: presencia/ausencia de deterioro cognoscitivo, tomando como referencia el punto de corte correspondiente al percentil 10 de la puntuación total en la prueba cognoscitiva aplicada por edad y escolaridad. Todos los sujetos con deterioro cognoscitivo que además presentaron dificultad en, por lo menos, una actividad instrumental de la vida diaria fueron clasificados en un tercer grupo: con deterioro cognoscitivo más dependencia funcional. Se calcularon las prevalencias de deterioro cognoscitivo y de deterioro cognoscitivo más dependencia funcional y se analizó la asociación con las variables sociodemográficas y de salud, mediante el análisis de regresión logística. Resultados. Del total de la población analizada, $7 \%$ tuvo deterioro cognoscitivo y $3.3 \%$ tuvo deterioro cognoscitivo más dependencia funcional. No se encontró asociación entre las variables sociodemográficas y de salud y la probabilidad de deterioro cognoscitivo; sin embargo, la probabilidad de deterioro cognoscitivo más dependencia funcional fue mayor en las mujeres, aumentó con la edad, fue menor en los sujetos casados y se asoció con la presencia de diabetes, enfermedad cerebral, enfermedad cardiaca y depresión. Conclusiones. La alta prevalencia del deterioro cognoscitivo en la población mexicana,y su relación con enfermedades crónicas frecuentes en la vejez, señala la importancia que tiene la identificación temprana de ambas condiciones en la población adulta mayor.

Palabras clave: envejecimiento; cognición; México
Mejía-Arango S, Miguel-Jaimes A,Villa A, Ruiz-Arregui L, Gutiérrez-Robledo LM.

Cognitive impairment and associated factors in older adults in Mexico.

Salud Publica Mex 2007;49 suppl 4:S475-S48I.

\begin{abstract}
Objective. To determine the prevalence of cognitive impairment in the elderly and its relation with sociodemographic and health factors in the Mexican population. Material and Methods. From the first wave data of the National Aging and Health Study, collected in $200 \mathrm{I}$ in Mexico, subjects 65 years or older and who had completed a cognitive assessment were selected. Subjects were classified according to two groups: with and without cognitive impairment, based on the cutoff point corresponding to the 10th percentile of the total cognitive assessment score, adjusted for age and educational level.All subjects with cognitive impairment that also had difficulty with one of the activities of daily living were classified as a third group: with cognitive impairment and functional dependency. Prevalence rates were calculated, and an association with demographic and health variables was established through regression analysis. Results. Seven percent of the population had cognitive impairment while $3.3 \%$ had both conditions. The probability of cognitive impairment was not significantly associated with any demographic or health variables. Cognitive impairment and functional dependency indicated an association with gender, age, marital status, diabetes, stroke, heart disease and depression. Conclusions. The high prevalence of cognitive impairment and its relation with chronic disease frequently observed in the Mexican elderly suggest the need to increase early identification of both conditions in the population.
\end{abstract}

Key words: aging; cognition; Mexico

(I) Instituto Nacional de Ciencias Médicas y Nutrición Salvador Zubirán. México.

Fecha de recibido: 22 de septiembre de 2005 • Fecha de aceptado: 3 I de enero de 2007

Solicitud de sobretiros: Dra. Silvia Mejía-Arango. Clínica de Geriatría. Instituto Nacional de Ciencias Médicas y Nutrición Salvador Zubirán. Vasco de Quiroga 15, col. Sección XVI. I4000 Tlalpan, México, DF, México.

Correo electrónico: silviamejiaa@hotmail.com 
E laumento en la expectativa de vida ha tenido implicaciones importantes para los sistemas de salud en el ámbito mundial. Las proyecciones señalan que, entre 1980 y el año 2050, la expectativa de vida para las personas mayores de 60 años aumentará $77 \% .{ }^{1}$ Con ello se incrementarán las enfermedades asociadas con la edad entre las que el deterioro cognoscitivo sin demencia y la demencia representan condiciones que afectan de manera directa la calidad de vida de la población adulta mayor y determinan un mayor uso de los servicios de salud. El deterioro cognoscitivo sin demencia constituye una condición de alto riesgo para la aparición de la demencia, si se considera que la probabilidad que tiene un individuo de desarrollarla es de 10 a 15\% anual, en comparación con sujetos de controles sanos, donde la conversión a demencia es de 1 a $2 \%$ anual. $^{2}$

En Latinoamérica, se han reportado tasas de prevalencia de demencia que oscilan entre 3 y $6 \%$ : Uruguay $(4.03 \%)$, Chile $(5.96 \%)$ y Brasil $(3.42 \%) .^{3-5}$ En la población mexicana se espera un crecimiento explosivo de la población geriátrica y, en consecuencia, un aumento significativo de casos de demencia. Actualmente se cuenta con algunos reportes clínicos sobre la presencia de $500 \mathrm{mil} \mathrm{a}$ 700 mil personas con demencia, de las cuales se estima que $25 \%$ no han sido diagnosticadas. ${ }^{6}$ Son pocos los reportes científicos sobre la prevalencia de la demencia en la población mexicana. En un estudio realizado en la Ciudad de México, ${ }^{7}$ se encontró una prevalencia de $4.7 \%$, mientras que en otro realizado en una comunidad de Jalisco, ${ }^{8}$ se reportó 3.3 por mil habitantes.

Algunas variables sociodemográficas que se han asociado con una mayor probabilidad de demencia son la edad, el género femenino y la baja escolaridad. ${ }^{9-11}$ Asimismo, algunas enfermedades crónicas de la vejez como diabetes, enfermedad pulmonar obstructiva, enfermedad cardiaca y cerebral, así como hipertensión, han mostrado una fuerte relación con el desarrollo de la demencia por sus efectos directos o indirectos en el estado del cerebro. ${ }^{12}$ Estas enfermedades, junto con la cirrosis, constituyen las seis principales causas de mortalidad en la población mexicana: enfermedades isquémicas del corazón $(14.9 \%)$, diabetes mellitus $(13.3 \%)$, enfermedad cerebro vascular $(8.6 \%)$, enfermedad pulmonar obstructiva (6.2\%) e hipertensión (3.5 por ciento). ${ }^{13}$

El análisis de los datos del Estudio Nacional de Salud y Envejecimiento en México permitirá examinar la importancia que tienen el deterioro cognoscitivo individual y el deterioro cognoscitivo más dependencia funcional en la población mexicana, así como la relación que ambos trastornos tienen con algunas enfermedades crónicas características del adulto mayor.

\section{Material y métodos}

El ENASEM es un estudio prospectivo tipo panel realizado en sujetos mexicanos nacidos antes de 1951, y en sus parejas. Fue realizado por el Centro de Estudios de Población de la Universidad de Pensilvania, el Centro de Investigaciones de Población de la Universidad de Maryland y el Centro de Demografía y Ecología de la Universidad de Wisconsin. ${ }^{14}$ El Instituto Nacional de Estadística, Geografía e Informática (INEGI) de México realizó el trabajo de campo. En el estudio se recoge información relacionada con diversos aspectos como son dinámica de la salud, estructura familiar y transferencias intergeneracionales, comportamientos migratorios y diferencias socioeconómicas según ingresos y posesión de bienes. Es representativo del componente no institucionalizado de la población mayor de 50 años en el año 2000. La recolección de los datos concluyó en el verano del 2001, y en el 2003 se realizó una segunda vuelta del estudio. En el 2001 participaron 15230 sujetos, los cuales representan a 13 millones de mexicanos. Los factores de ponderación se definieron de acuerdo con cuatro criterios: a) por hogar, b) por sujeto de 50 años o más, con pareja de 50 años o más en el mismo hogar, c) por sujetos de 50 años o más, sin pareja, y d) por sujetos menores de 50 años, con pareja de 50 años o más en el mismo hogar.

Para la selección de la muestra se analizó el archivo de cognición de las bases de datos del ENASEM, el cual contiene información de 14129 sujetos. Se seleccionaron todos los que cumplieran con los siguientes criterios: a) ser mayores de 65 años; b) que hubieran completado la evaluación cognoscitiva (que no tuvieran un déficit sensorial que impidiera la realización de la prueba), y c) que hubieran reportado el número de años de estudio. El número total de sujetos que conformaron la muestra fue de 4 182. Para todos los sujetos que participaron en el estudio se obtuvo el consentimiento informado de acuerdo con lo que establecen los "Principios éticos para las investigaciones médicas en seres humanos" de la Declaración de Helsinki.

Se construyeron dos variables dependientes: presencia/ ausencia de deterioro cognoscitivo y presencia/ ausencia de deterioro cognoscitivo más dependencia funcional. Para la clasificación del deterioro cognoscitivo se tomó como referencia la variable que contenía la puntuación total en la prueba cognoscitiva aplicada en el estudio (versión reducida del Examen Cognoscitivo Transcultural [Cross-Cultural Cognitive Examination]). Esta prueba tiene una puntuación máxima de 80 puntos e incluye la evaluación de las siguientes funciones cognoscitivas: a) memoria verbal primaria (repetición 
de ocho palabras; b) atención selectiva (detección visual de un estímulo blanco); c) memoria verbal secundaria (evocación de las ocho palabras); d) praxias visoconstruccionales (copia de dos figuras geométricas); e) memoria visual (evocación de las figuras geométricas). Con base en el diagnóstico clínico neurológico de un grupo de sujetos con demencia y sin demencia, se calculó la sensibilidad (100\%) y la especificidad (83\%) de la versión reducida. ${ }^{15}$ Se analizó la distribución percentilar de la puntuación total en la prueba dividiendo a los sujetos en cuatro rangos de escolaridad (0, 1-4, 5-9 y 10 años o más) dentro de tres rangos de edad (65-74, 75-84 y 85 años y más). Se tomaron los puntos de corte correspondientes al percentil 10 dentro de cada uno de los 12 estratos (edad x escolaridad) y se clasificó con deterioro cognoscitivo a todos los sujetos por debajo del punto de corte correspondiente.

Para la clasificación de los sujetos con alteración funcional se tomaron las variables que registraban la necesidad de ayuda para realizar alguna de las siguientes actividades instrumentales de la vida diaria (AIVD): a) manejar dinero, $b$ ) hacer compras solo, c) preparar una comida, d) administrar sus medicamentos. Se encontró que $38.9 \%$ de los sujetos no requerían ayuda para realizar las AIVD; $22.5 \%$ la requerían para llevar a cabo una AIVD; $20.2 \%$ la requerían para dos; $7.8 \%$, para tres, y $10.6 \%$, para el total de las AIVD consideradas.

Se seleccionaron todos los sujetos que, por lo menos, requerían ayuda en una AIVD, y en combinación con la variable de deterioro cognoscitivo se clasificaron en cuatro grupos: a) sin alteración cognoscitiva y sin alteración funcional, b) con alteración funcional, c) con alteración cognoscitiva y d) con alteración cognoscitiva más alteración funcional. Para el análisis posterior se compararon los siguientes grupos: a) sin alteración cognoscitiva y sin alteración funcional, b) con alteración cognoscitiva y c) con alteración cognoscitiva más alteración funcional.

Como variables independientes se seleccionaron dos grupos de variables:

1. Variables demográficas: género, edad, escolaridad, estado civil y tipo de ocupación, que a su vez fueron categorizadas en tres estratos de edad (65-74, 75-84 y 85 años y más), cuatro niveles de escolaridad ( 0 , 1-4, 5-9 y 10 años o más), y cuatro categorías de estado civil (soltero, casado, divorciado-separado, viudo).

2. Variables de salud: se seleccionaron las que registró el autorreporte de enfermedad: hipertensión o presión alta, diabetes o nivel de azúcar alto en sangre, cáncer, enfermedad pulmonar, enfermedad cardíaca (ataque al corazón), enfermedad cerebral (embolia, derrame cerebral o isquemia cerebral transitoria). Para su registro, los sujetos respondieron a la pregunta sobre presencia/ausencia de diagnóstico médico de las diferentes enfermedades. También se incluyó la variable depresión que corresponde a la puntuación total (0-9) obtenida por cada sujeto en un cuestionario de nueve preguntas sobre síntomas depresivos.

Para el análisis de los datos se calculó la prevalencia de deterioro cognoscitivo y de deterioro cognoscitivo más dependencia funcional en la muestra total, y en la muestra estratificada por sexo, edad y escolaridad. Se realizó un análisis univariado para determinar la distribución de las variables independientes en cada grupo. Finalmente se calculó un modelo multivariado con todas las variables independientes, por medio de la regresión logística por pasos (stepwise) en cada uno de los grupos.

\section{Resultados}

Se analizó un total de 4183 sujetos adultos mayores del ENASEM. La proporción de mujeres es un poco mayor que la de hombres. Las edades se encuentran en un rango de 65 a 105 años, con una edad promedio de 72.5 años ( $\mathrm{DE}=6.3$ ). La mayoría de los sujetos tiene entre 65 y 74 años; aproximadamente $30 \%$ tiene entre 75 y 84 años, y sólo una proporción pequeña (5.7\%) tiene más de 85 años. La escolaridad promedio fue de 3.3 años ( $\mathrm{DE}=3.8$ ) con un rango entre 0 y 19 años de estudio. De la muestra, $70 \%$ es analfabeta o tiene menos de cinco años de escolaridad. El estado civil está caracterizado por una mayor proporción de sujetos casados y viudos (cuadro I). En el cuadro II pueden observarse las prevalencias totales por sexo, edad y escolaridad en los dos grupos de sujetos: con deterioro cognoscitivo y con deterioro cognoscitivo más dependencia funcional. La prevalencia del primero en los sujetos sin dependencia funcional es de 7.1\% (IC95\% 0.063-0.078). No se observan diferencias por género, edad, escolaridad y estado civil, ni un cambio en la probabilidad de deterioro asociado con estas variables (cuadro III). Ninguna de las comorbilidades autorreportadas mostró asociación significativa con el deterioro cognoscitivo (cuadro IV).

La prevalencia de deterioro cognoscitivo más dependencia funcional es de 3.3\% (IC95\% 0.027-0.038). $\mathrm{El}$ análisis univariado muestra diferencias por género, edad y estado civil según puede observarse en el cuadro II. La probabilidad es mayor en las mujeres; aumenta con la edad, y es menor en sujetos casados (cuadro III). En los sujetos con autorreporte de diabetes, enfermedad pulmonar obstructiva, enfermedad cardiaca, 
Cuadro I

Características demográficas de adultos mayores DE 65 AÑos. MÉXICO, 200 I

\begin{tabular}{lc} 
Total & 4183 \\
\hline $\begin{array}{l}\text { Sexo } \\
\text { Masculino }\end{array}$ & 47.3 \\
\hline Femenino & 52.7 \\
\hline Edad & 66.7 \\
65-74 años & 27.6 \\
\hline 75-84 años & 5.7 \\
\hline 85 años o más & \\
Nivel educativo & 34.4 \\
\hline años & 36 \\
\hline I-4 años & 24 \\
\hline 5-9 años & 5.6 \\
\hline I0 años o más & \\
Estado civil & 3.8 \\
\hline Soltero & 57 \\
\hline Casado & 6.7 \\
\hline Divorciado/separado & 32.5 \\
\hline Viudo &
\end{tabular}

Cuadro II

Prevalencia de deterioro cognoscitivo y de DETERIORO COGNOSCITIVO CON DEPENDENCIA FUNCIONAL de adultos mayores de 65 años. MÉxico, 200 I

\begin{tabular}{|c|c|c|c|c|}
\hline & \multicolumn{2}{|c|}{ Deterioro cognoscitivo } & \multicolumn{2}{|c|}{$\begin{array}{l}\text { Deterioro cognoscitivo } \\
\text { más dependencia } \\
\text { funcional }\end{array}$} \\
\hline & & p & & p \\
\hline TOTAL (\%) & 7.1 & & 3.3 & \\
\hline \multicolumn{5}{|l|}{ Sexo (\%) } \\
\hline Masculino & 8.9 & NS & 3.0 & 0.003 \\
\hline Femenino & 7.7 & & 5.0 & \\
\hline \multicolumn{5}{|l|}{ Edad (\%) } \\
\hline 65-74 años & 8.5 & NS & 3.2 & 0.002 \\
\hline $75-84$ años & 7.2 & & 5.5 & \\
\hline 85 años o más & 11.3 & & 7 & \\
\hline \multicolumn{5}{|c|}{ Nivel educativo (\%) } \\
\hline 0 años & 8.7 & NS & 4.8 & NS \\
\hline I-4 años & 9 & & 3.7 & \\
\hline 5-9 años & 7.3 & & 3.1 & \\
\hline 10 años o más & 5.7 & & 4.8 & \\
\hline \multicolumn{5}{|l|}{ Estado civil (\%) } \\
\hline Soltero & 7.8 & NS & 6.5 & 0.016 \\
\hline Casado & 7.8 & & 3.2 & \\
\hline \multicolumn{2}{|c|}{ Divorciado/separado 9.6} & & 4.4 & \\
\hline Viudo & 8.9 & & 5.1 & \\
\hline
\end{tabular}

\section{Cuadro III \\ REGRESIÓN LOGísTICA DE VARIABLES SOCIODEMOGRÁFICAS De adultos mayores de 65 años. México, 2001}

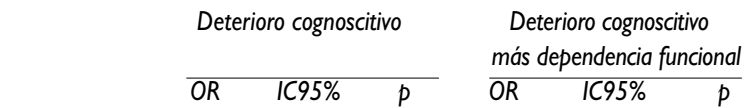

Sexo

\begin{tabular}{lllllll} 
Masculino & $\mathrm{I}$ & & $\mathrm{I}$ & & \\
\hline Femenino & 0.85 & $0.66-1.07$ & 0.17 & $\mathrm{I} .68$ & $\mathrm{I} .18-2.40$ & 0.004
\end{tabular}

Edad

\begin{tabular}{lllllll}
$65-74$ años & I & \multicolumn{1}{l}{} \\
\hline $75-84$ años & 0.83 & $0.62-1.10$ & 0.90 & 1.73 & $1.20-2.48$ & 0.003
\end{tabular}

$\begin{array}{lllllll}85 \text { años o más } & \text { I.37 } & 0.81-2.31 & 0.66 & 2.24 & \mathrm{I} .13-4.43 & 0.020\end{array}$

Nivel educativo

\begin{tabular}{lllllll}
0 años & 1.58 & $0.85-2.92$ & 0.14 & 1.00 & $0.50-2.0$ & 0.99 \\
\hline $1-4$ años & 1.62 & $0.87-2.99$ & 0.12 & 0.76 & $0.38-1.54$ & 0.45 \\
\hline
\end{tabular}

\begin{tabular}{lllllll}
\hline $\mathrm{I}-4$ años & $\mathrm{I} .62$ & $0.87-2.99$ & 0.12 & 0.76 & $0.38-\mathrm{I} .54$ & 0.45 \\
\hline
\end{tabular}

\begin{tabular}{llllllll}
\hline $5-9$ años & 1.29 & $0.69-2.44$ & 0.41 & 0.63 & $0.30-1.34$ & 0.23 \\
\hline
\end{tabular}

10 años o más I

Estado civil

Soltero

\begin{tabular}{lllllll}
\hline Casado & I.0I & $0.52-1.96$ & 0.97 & 0.43 & $0.21-0.84$ & 0.023 \\
\hline
\end{tabular}

\begin{tabular}{lllllll}
\hline Divorciado & 1.26 & $0.58-2.73$ & 0.55 & 0.60 & $0.24-1.54$ & 0.29 \\
\hline Viudo & 1.16 & $0.59-2.29$ & 0.65 & 0.71 & $0.34-1.48$ & 0.36
\end{tabular}

Viudo

Cuadro IV

Regresión logística de VARIABLES

DE COMORBILIDAD DE ADULTOS MAYORES DE 65 AÑOS. MÉxico, 200I

\begin{tabular}{|c|c|c|c|c|c|c|}
\hline & \multicolumn{3}{|c|}{ Deterioro cognoscitivo } & \multicolumn{3}{|c|}{$\begin{array}{l}\text { Deterioro cognoscitivo } \\
\text { más dependencia funcional }\end{array}$} \\
\hline & $O R$ & IC95\% & $p$ & $O R$ & IC95\% & $p$ \\
\hline & & & & & & \\
\hline Hipertensión & 0.84 & $0.62-1.15$ & 0.29 & 1.45 & $0.95-2.21$ & 0.07 \\
\hline Diabetes & 1.5 & $0.79-1.71$ & 0.44 & 2.42 & $1.54-3.80$ & 0.0001 \\
\hline Cáncer & 0.39 & $0.05-2.86$ & 0.35 & 0.78 & $0.10-5.84$ & 0.81 \\
\hline Enf. pulmonar & 0.18 & $0.31-1$. & 0.63 & 2.03 & I.10-3.74 & 0.02 \\
\hline Enf. cardiaca & 1.37 & $0.72-2.62$ & 0.33 & 2.65 & I.33-5.29 & 0.005 \\
\hline Enf. cerebral & 1.36 & $0.61-3.03$ & 0.45 & 5.69 & $2.99-10.81$ & 0.0001 \\
\hline Depresión & 1.04 & $0.98-1.10$ & 0.19 & 1.33 & I.22-I.44 & 0.0001 \\
\hline
\end{tabular}

enfermedad cerebral y depresión, la probabilidad de deterioro cognoscitivo más dependencia funcional es significativamente mayor (cuadro IV).

El resultado del análisis multivariado muestra que el modelo que explica la presencia de deterioro cognoscitivo más dependencia funcional está conformado por el género femenino, mayor edad y las comorbilidades: diabetes, enfermedad cerebral y depresión (cuadro V). 


\section{Cuadro V}

Modelo de REgRESIÓN LOGísticA CON LOS FACTORES ASOCIADOS Y EL DETERIORO COGNOSCITIVO MÁS DEPENDENCIA FUNCIONAL DE ADULTOS MAYORES DE 65 AÑos. MÉxICO, 200 I

\begin{tabular}{|c|c|c|c|}
\hline & $O R$ & $I C 95 \%$ & $p$ \\
\hline \multicolumn{4}{|l|}{ Sexo } \\
\hline Masculino & $\mathrm{I}$ & & \\
\hline Femenino & 1.62 & $1.01-2.59$ & 0.043 \\
\hline \multicolumn{4}{|l|}{ Edad } \\
\hline $65-74$ años & 1 & & \\
\hline 75-84 años & 1.80 & I.I3-2.87 & 0.014 \\
\hline 85 años o más & 3.29 & I.44-7.47 & 0.004 \\
\hline \multicolumn{4}{|l|}{ Salud } \\
\hline Diabetes & 2.07 & I.28-3.34 & 0.003 \\
\hline Enfermedad cerebral & 5.85 & $2.86-11.94$ & 0.0001 \\
\hline Depresión & 1.27 & I.I7-1.39 & 0.0001 \\
\hline
\end{tabular}

\section{Conclusiones}

Los resultados sugieren que el deterioro cognoscitivo es una condición importante en la población de adultos mayores mexicanos. La prevalencia de $7 \%$ encontrada en nuestro estudio es similar a la reportada por otros. ${ }^{16-17}$ Para ambas condiciones (deterioro cognoscitivo más dependencia funcional), la prevalencia fue de $3.3 \%$, similar a la reportada para demencia en algunos países de Latinoamérica. ${ }^{3-5}$ Aunque en el presente estudio no se emplearon criterios clínicos para clasificar a los sujetos con demencia, la presencia de deterioro cognoscitivo más dependencia funcional constituyen dos de los criterios principales para el diagnóstico de demencia, de acuerdo con los sistemas de clasificación más empleados. ${ }^{18}$

En relación con la escolaridad, frecuentemente se ha reportado que tanto la prevalencia como la incidencia del deterioro cognoscitivo es mayor en sujetos con bajo logro educativo. ${ }^{9-11}$ La mayoría de los estudios que reportan este efecto emplean puntos de corte únicos para la clasificación de los sujetos con deterioro cognoscitivo. Generalmente son puntos de corte que provienen de estudios hechos en poblaciones con pocas variaciones en la escolaridad. Sin embargo, en poblaciones con bajo nivel escolar, como la población mexicana, la aplicación de estos puntos de corte da como resultado una sobreestimación del deterioro cognoscitivo. ${ }^{19}$ El cálculo de las prevalencias de deterioro cognoscitivo con los puntos de corte únicos en sujetos analfabetas considerados en diferentes estudios, incluyendo el ENASEM, ${ }^{20}$ son desproporcionadamente altas; clasifican con deterioro a sujetos con bajas puntuaciones en las pruebas cognoscitivas, lo cual está determinado principalmente por el efecto de la baja escolaridad sobre el desempeño en las pruebas, ${ }^{21}$ más que por un déficit cognoscitivo. En consecuencia, en el presente estudio se aplicó la metodología de percentiles, ajustando los puntos de corte por edad y escolaridad, sin tomar en cuenta el efecto de esta última en el deterioro cognoscitivo.

En el grupo de sujetos con deterioro cognoscitivo más dependencia funcional, la probabilidad es mayor en las mujeres, aumenta con la edad y es menor en los sujetos casados. Estos resultados coinciden con los reportados por diversos estudios ${ }^{17,22-23}$ llevados a cabo en Latinoamérica, Estados Unidos y Europa. En relación con las variables de salud, se encontró que la probabilidad de deterioro cognoscitivo más dependencia funcional era mayor en sujetos con autorreporte de diabetes, enfermedad pulmonar obstructiva, enfermedad cardiaca, enfermedad cerebral y depresión. El peso significativo de la diabetes, enfermedad pulmonar obstructiva, enfermedad cardiaca, enfermedad cerebral y depresión en forma individual y conjunta (diabetes, enfermedad cerebral y depresión), dentro de un modelo con la edad y el género, coincide con algunos estudios. La alta prevalencia de diabetes y enfermedad cerebral en pacientes con demencia ha sido reportada en poblaciones méxico-americanas. ${ }^{23}$ Consideradas como seis de las primeras causas de mortalidad en la población mexicana, ${ }^{13}$ la diabetes, enfermedad cerebral, enfermedad cardiaca y enfermedad pulmonar obstructiva representan enfermedades crónicas importantes durante la vejez, cuyos efectos micro y macrovasculares sobre el cerebro, ${ }^{12}$ pueden señalar un aumento en el riesgo de demencia. El peso mayor de algunas comorbilidades como la diabetes en la población méxico-americana ha llevado a algunos autores a plantear que, aunque las cifras de prevalencia de demencia son similares, la etiología puede ser diferente al de otras poblaciones, donde el peso de factores genéticos es mayor. ${ }^{23}$ De igual manera, se ha reportado una alta prevalencia de depresión en la población mexicana. Las cifras van desde 10 hasta $40 \%$, dependiendo del tipo de población estudiada y del instrumento de clasificación empleado. ${ }^{24-26} \mathrm{Su}$ fuerte asociación con la demencia ha sido también una evidencia ampliamente reportada en diversos estudios, en los que se plantea el papel de la depresión como síntoma preclínico y asociado a la demencia. ${ }^{27-28}$

En el grupo de sujetos que presentan deterioro cognoscitivo sin alteración funcional, no se observaron asociaciones significativas con variables sociodemográficas y de salud. Este grupo es similar al descrito por 
varios autores como sujetos con "deterioro cognoscitivo sin demencia", en el que se ha reportado una alta heterogeneidad tanto en su presentación clínica como en su etiología..$^{29-32}$ La ausencia de un patrón típico de la demencia sugiere que este grupo es altamente heterogéneo, tanto en la distribución de las variables sociodemográficas como en la presencia de algunas comorbilidades frecuentemente asociadas con la demencia. La aparición de alteraciones en actividades de la vida diaria y la asociación con algunas comorbilidades constituyen características que diferencian a los dos grupos analizados. Su presencia podría determinar el alto riesgo de convertirse en demencia que tiene un subgrupo de sujetos, mientras que otro podría estar representando un subtipo con baja probabilidad de conversión. Sin embargo este resultado solo podrá considerarse una vez que se analice la evolución de los sujetos.

La clasificación del deterioro cognoscitivo por medio de la metodología de los percentiles, y el uso de información autorreportada sobre la presencia de algunas enfermedades, constituyen limitaciones del presente estudio, si se comparan con los procedimientos de diagnóstico clínico empleados en otras investigaciones. Sin embargo, en estudios poblacionales como el presente, diseñados para recoger una amplia diversidad de datos sobre la salud y el envejecimiento, no es posible aplicar procedimientos estándar de diagnóstico clínico, y es recomendable el uso de estos métodos. ${ }^{33} \mathrm{~A}$ pesar de lo anterior, los resultados de nuestra investigación representan un aporte valioso para la comprensión de la importancia que tiene el deterioro cognoscitivo y la demencia en la población mexicana, así como su asociación con enfermedades altamente prevalentes en los adultos mayores de México.

\section{Referencias}

I. Banco Mundial. Informe sobre el desarrollo mundial 1993. Invertir en salud. Washington: Oxford University Press, 1994.

2. Petersen RC, Doody R, Kurz A, Mohs R, Morris JC, Rabins P, et al. Current concepts in mild cognitive Impairment.Arch Neurol 200 I;58:1985-1992.

3. Ketzoian C, Romero S, Dieguez E, Cairolo G, Rega Y, Caseres R, et al.

Prevalence of demential sindromes in a population of Uruguay. Study of "Villa del Cerro".J Neurol Sci 1997;150-I55.

4. Quiroga P. Dementia Prevalence in Concepción Chile. Dementia Project-WHO-Chile. World Congress of Geriatrics and Gerontology: 1997.Adelaida,Australia.

5. Herrera E, Caramelli P, Silveira AS, Mathias SC, Nitrini R. Population epidemiology survey of dementia in Catanduva, Brazil. Preliminary Results. J Neurol Sci 1997;150:155-156.

6. Navarrete H, Rodríguez-Leyva I. La demencia. ¿Subdiagnosticada o ignorada? Rev Mex de Neurociencias 2003;4:I I-I2.
7. Gutiérrez LM, Ostrosky F, Sanchez S,Villa A. Prevalence of dementia and mild cognitive impairment in subjects 65 years older in Mexico City: An Epidemiological Survey. Gerontology 2001;47:145.

8. Cruz-Alcalá L. Prevalencia de algunas enfermedades neurológicas en la Ciudad de Tepatitlán, Jalisco. Rev Mex de Neurociencias 2002;3:7I-76. 9. Fabrigoule C, Letteneur L, Dartigues JF, Zarrouk M, Commenges D, Barberger-Gateau P. Social and leisure activities and risk of dementia:A prospective longitudinal study. Journal of the American Geriatric Society 1995;43:485-490.

10. Bonaiuto S, Rocca WA, Lippi A, Giannandrea E, Mele M, Cavarzeran F, et al. Education and occupation as risk factors for dementia: a populationbased case-control study. Neuroepidemiology 1995; I 4(3): I0I-I09.

II. Scarmeas N, Levy G, Tang MX, Manly J, Stern Y. Influence of leisure activity on the incidence of Alzheimer's Disease. Neurology 2001;57:2236-2242. 12. Whitmer RA, Sydney S, Selby J, Claiborne-Johnston S, Yaffe K. Midlife cardiovascular risk factors and risk of dementia in late life. Neurology 2005; 64:277-28I.

13. Instituto Nacional de Estadística, Geografía e Informática. Conteo de población y vivienda 2000. Resultados definitivos nacionales. México, DF: INEGl; 2000. Disponible en: http//www.inegi.gob.mx.

14. Palloni A, Soldo B,Wong R. Health status in a nacional sample ofelderly Mexicans. Paper prepared for presentation at the Gerontological Society of America Conference; noviembre, 2002. Boston, EUA.

15. Glosser G,Wolfe N,Albert ML, Lavine L, Steele JC, Calne DB, et al. Cross-cultural cognitive examination:Validation of a dementia screening instrument for neuroepidemiological research. J Am Geriatr Soc 1993;41:931-939.

16. Di Carlo A, Balderaschi M,Amaducci L. Factores de riesgo y de protección en la enfermedad de Alzheimer. En: Mangone CA,Allegri R F,Arizaga RL, Ollarri JA, eds. Enfoque multidisciplinario. Buenos Aires: Sagitario, 1997.

17. Unverzagt FW, Gao S, Baiyewu O, Ogunniyi AO, Gureje O, Perkins A, et al. Prevalence of cognitive impairment: data from the Indianapolis Study of Health and Aging. Neurology 200 I;57: 1655-1662.

18. American Psychiatric Association: Diagnostic and statistical manual of mental sisorders, 4th edition. Washington, DC:American Psychiatric Association, 1994.

19. Mejía S, Gutierrez LM,Villa A, Ostrosky-Solis F. Cognition, functional status, education and the diagnosis of dementia and mild cognitive impairment in Spanish speaking elderly.Appl Neuropsychol 2004; I I:196-203. 20. Gutiérrez LM, Mejía S, Miguel A, Villa A, Ostrosky F. Uso de metodologías conocidas y desarrollo de nuevas herramientas para la evaluación internacional y comparativa de la salud y el bienestar de los adultos mayores. Compatibilización, estandarización y validación de instrumentos existentes para detectar deficiencias cognitivas entre adultos mayores hispanohablantes; 2004. Proyecto Organización Panamericana de la Salud. (www.paho.org)

21. Ardila A, Ostrosky-Solis F, Rosselli M, Gómez C.Age-related cognitive decline during normal aging: the complex effect of education. Arch Clin Neuropsychol 2000;5:495-513.

22. Launer LJ,Andersen K, Dewey ME, Letenneur L, Ott A,Amaducci LA, et al. Neurology 1999;52: 78-84

23. Nguyen HT, Black SA, Ray LA, Espino DV, Markides KS. Predictors of decline in MMSE scores among older Mexican-Americans.J Gerontol: Medical Sciences 2002;57:181-185.

24. Reyes S. Population ageing in the Mexican Institute of Social Security. Health policy and economic implications. México: IMSS-Fundación Mexicana para la Salud, 200I.

25. García-Peña C. Unidad de Investigación Epidemiológica y en Servicios de Salud/Area Envejecimiento. México DF: Centro Médico Nacional XXI/Instituto Mexicano del Seguro Social, 2006. 
26. Ocegueda-Azpeitia G, Pichardo-Fuster A. Prevalencia de la depresión en ancianos mexicanos. Arch Geriátrico 2003;6:78-8I.

27. Mejia S, Giraldo M, Pineda D,Ardila A, Lopera F. Nongenetic factors as modifiers of age of onset of familial Alzheimer's disease. Int Psychogeriatr 2003; 15:337-349.

28. Jorm AF. Is depression a risk factor for dementia or cognitive decline? Neurobiol Aging 2000;2I(SupplI): S215.

29. Ritchie K,Artero S, Touchon J. Classification criteria for mild cognitive impairment:A population-based validation study. Neurology 200I;56:37-42. 30. Lopez OL, Jagust WJ, DeKosky ST, Becker JT, Fitzpatrick A, Dulberg

$\mathrm{C}$, et al. Prevalence and classification of mild cognitive impairment in the Cardiovascular Health Study. Cognition study. Arch Neurol 2003;60:1385-1389.

31. Unverzagt FW, Gao S, Baiyewu O, Ogunniyi AO, Gureje O, Perkins A, , et al. Prevalence of cognitive impairment: data from the Indianapolis Study of Health and Aging. Neurology 2003; 13(57): 1655- 1662.

32. Petersen RC, Morris JC. Clinical features. In: Petersen RC ed. Mild cognitive impairment:Aging to Alzheimer's disease. New York: Oxford University Press, 2003.

33. Feinstein AL. Principles of Medical statistics. Boca Raton, Florida: Chapman \& Hall/CRC, 2002. 